\title{
Channel Modeling for UAV-based Optical Wireless Links with Nonzero Boresight Pointing Errors
}

\author{
Mohammad Taghi Dabiri, Mohsen Rezaee, and Imran Shafique Ansari
}

\begin{abstract}
The channel modeling of unnamed aerial vehicle (UAV)-based free-space optical (FSO) links with nonzero boresight pointing error is the subject of this paper. In particular, utilizing log-normal turbulence model, we propose a novel closedform statistical channel model for UAV-based FSO links that takes into account the effect of nonzero boresight pointing errors. Subsequently, utilizing Gamma-Gamma turbulence model, we propose a novel channel characterization for such links that is valid under moderate to strong turbulence conditions. The accuracy of the proposed models is verified via Monte-Carlo simulations. The proposed models are more tractable and suitable for analysis of such UAV-based FSO links.
\end{abstract}

Index Terms-Angle-of-arrival fluctuations; free-space optics; nonzero boresight pointing error, unmanned aerial vehicles.

\section{INTRODUCTION}

$\mathbf{T}$ HE realization of reliable free-space optical (FSO) backhaul and fronthaul communication links between unmanned aerial vehicles (UAVs) acting as aerial base stations is a milestone for the future development of communication networks [1]-[3]. Although channel modeling in the context of terrestrial FSO communications has been studied in [4][7], these studies cannot be directly used for UAV-based FSO systems. There are several works in the literature of the longrange optical communications in space and stratosphere [8], [9]. However, there is one main difference between longrange optical communication and short-range multi rotor UAVbased optical communications. In all of the proposed works in the context of optical space communications, the link length is assumed to be in the order of several hundred to several thousand kilometers, and thus, the standard deviation of alignment error must be in the order of $\mu \mathrm{rad}$. For instance, in [8], the value of standard deviation of misalignment is 100 $\mu$ rad for a $100 \mathrm{~km}$ stratosphere link length and in [9], the value of standard deviation of misalignment is $0.05 \mu \mathrm{rad}$ for a 200 $\mathrm{km}$ stratosphere link length. As a result, for establishing such communication links, we have to use fast and precise stabilizers which are bulky and very expensive. However, due to the payload and power consumption limitations of lightweight quadcopters drones, reaching such alignment accuracy in the order of $\mu \mathrm{rad}$ may not be always possible. From the literature of short range optical communications (the link length is mainly in the order of a few hundred meters), the standard deviation of AoA fluctuations due to orientation fluctuations of lightweight multi-rotor drones is in the order of several mrad which is approximately 250-1000 times larger than the standard deviation of AoA for the space as well as the ground FOS links [10], [11]. To assess the benefit of short-range multi rotor UAV-based optical communications, one important aspect is to accurately model the channel, which has been the subject of a few recent works [12]-[17]. In [12], [13], a novel model was presented for FSO link between two hovering UAVs with multi-element optical transceiver arrays. In [14], [15], the authors derived a statistical model for UAV-based FSO system by taking into account the non-orthogonality of the laser beam and the random fluctuations of UAVs. Considering the joint effects of UAVs' fluctuations as well as atmospheric turbulence, a novel channel model was proposed in [16], [17] that is suitable for hovering UAV-based FSO links with zero boresight angle. However, none of the proir studies addresses the effects of nonzero boresight UAV's angle. Moreover, the results of [12]-[17] are obtained for a special case wherein the UAVs have equal variances of orientation fluctuations in $x-z$ and $y-z$ axes.

In addition to the UAVs' position and orientation fluctuations, in practical situations, inevitable errors such as position estimation errors and mechanical noise lead to a fixed misalignment between transmitter ( $\mathrm{Tx})$ and receiver $(\mathrm{Rx})$ mounted on UAVs, which is known as boresight. More importantly, the variances of orientation fluctuations are not equal in $x-z$ and $y-z$ axes. Hence, to assess the advantages of UAV-based FSO systems, in this paper, we consider a general case wherein the variances of UAV oientation fluctuations in $x-z$ and $y-z$ axes can take any different values and we propose a comprehensive and accurate channel model by taking into account the effects of nonzero boresight. In particular, under the weak turbulence conditions, we propose a novel and tractable channel model for the considered UAV-based FSO link over log-normal atmospheric turbulence environment that takes into account the effects of nonzero boresight as well as UAVs' orientation and position fluctuations, atmospheric turbulence strength, optical beamwidth, link length, lens radius size, receiver's field-ofview (FOV), etc. For moderate to strong turbulence conditions, a novel closed-form statistical channel model is derived under the influence of Gamma-Gamma (GG) turbulence channel. The accuracy of the proposed models is verified by performing Monte-Carlo simulations. The developed results can therefore be applied as a benchmark for determining the optimal tunable parameters of UAV-based FSO links under different channel conditions and varying levels of UAV instability without resorting to time-consuming Monte-Carlo simulations.

\section{System Model AND MAIN Assumptions}

Similar to [16], [17], we consider a UAV-based FSO system wherein a hovering UAV Tx (located at $[0,0,0]$ in Cartesian coordinate system $[x, y, z])$ transmits optical signals towards a 
hovering UAV Rx (located at $[0,0, Z]$ ). By knowing the mean positions of $\mathrm{Tx}$ and Rx, the UAVs try to align Tx and Rx. However, due to the inherent position and orientation fluctuations of UAVs, the instantaneous position and orientation of aerial nodes are deviated from their means. Let the random variables $(\mathrm{RVs}) \theta_{\mathrm{tx}}$ and $\theta_{\mathrm{ty}}$ denote the orientation fluctuations of Tx in $x-z$ and $y-z$ planes, respectively, the RVs $\theta_{\mathrm{rx}}$ and $\theta_{\text {ry }}$ denote the orientation fluctuations of $\operatorname{Rx}$ in $x-z$ and $y-z$ planes, respectively, the RVs $x_{t x}$ and $y_{t y}$ denote the position vibrations of Tx in $x-z$ and $y-z$ planes, respectively, and the RVs $x_{r x}$ and $y_{r y}$ denote the position vibrations of $\mathrm{Rx}$ in $x-z$ and $y-z$ planes, respectively. Based on numerous random events related to hovering UAVs and from the central limit theorem, position and orientation deviations of UAVs are considered as Gaussian distributed [12], [18], [19].

In practical situations, in addition to aforementioned UAV's fluctuations, position estimation errors and mechanical noise lead to a fixed displacement in UAVs' orientation, which is termed as boresight. Therefore, we have $\theta_{\mathrm{i}} \sim \mathcal{N}\left(\theta_{\mathrm{i}}^{\prime}, \sigma_{i o}^{2}\right)$ for $i \in\{\mathrm{tx}, \mathrm{ty}\}, \theta_{\mathrm{i}} \sim \mathcal{N}\left(\theta_{\mathrm{i}}^{\prime}, \sigma_{i o}^{2}\right)$ for $i \in\{\mathrm{rx}, \mathrm{ry}\}, x_{i} \sim \mathcal{N}\left(0, \sigma_{i p}^{2}\right)$ for $i \in\{\mathrm{tx}, \mathrm{rx}\}$, and $y_{i} \sim \mathcal{N}\left(0, \sigma_{i p}^{2}\right)$ for $i \in\{\mathrm{ty}, \mathrm{ry}\}$. From these, $r_{d}$ is the radial distance between the received beam center and the Rx lens center where

$$
\begin{aligned}
& r_{d}= \\
& \sqrt{\left(Z \tan \left(\theta_{t x}\right)+x_{t x}+x_{r x}\right)^{2}+\left(Z \tan \left(\theta_{t y}\right)+x_{t y}+x_{r y}\right)^{2}} .
\end{aligned}
$$

The optical channel model between UAVs can be formulated as

$$
h=h_{l} h_{a} h_{p g} h_{p a},
$$

where $h_{l}$ is the channel loss, $h_{a}$ is the atmospheric turbulence, $h_{p g}$ is the geometrical loss due to the deviation between the received beam center and the receiver lens center, and $h_{p a}$ is the link loss induced by the angle-of-arrival (AoA) fluctuation.

From (1) and [4, eq. (8)], for any instantaneous value of $r_{d}$, the instantaneous collected optical signal by a $\mathrm{Rx}$ lens with radius $r_{a}$ (which is called geometrical pointing error coefficient) can be obtained as

$$
\begin{aligned}
h_{p g}= & \int_{-r_{a}}^{r_{a}} \int_{-\sqrt{r_{a}^{2}-y^{2}}}^{\sqrt{r_{a}^{2}-y^{2}}} \frac{2}{\pi w_{z}^{2}} \\
& \times e^{-2 \frac{\left(x+\theta_{t x} Z+x_{t x}+x_{r x}\right)^{2}+\left(y+\theta_{t y} Z+x_{t y}+x_{r y}\right)^{2}}{w_{z}^{2}}} d x d y .
\end{aligned}
$$

In addition, the AoA of the received signal is obtained as

$$
\theta_{a}=\tan ^{-1}\left(\sqrt{\left(\tan \left(\theta_{t x}+\theta_{r x}\right)\right)^{2}+\left(\tan \left(\theta_{t y}+\theta_{r y}\right)\right)^{2}}\right) .
$$

As depicted in Fig. 11 the collected optical signal by the converging $\mathrm{Rx}$ lens is guided toward a circular detector with radius $r_{a p}$. When an incident beam with small value of $\theta_{a}$ is passed through a lens, the outside angle of beam will be approximately unaltered [20]. As shown, a thin lens diffracts the collected light into a series of circular waves at the focal plane. The intensity of the diffracted beam pattern at the focal plane can be expressed by using the Airy pattern which is given in [21]. The fraction of collected power by the circular detector to the total power collected by the lens is the link loss induced by the AoA fluctuation which is denoted by $h_{p a}$. From [21] and [8], for the considered system model, $h_{p a}$ can be obtained from (5). In (5), $d_{f}$ is the focal length, $N_{f}$ is the f-number, $\lambda$ is the optical wavelength, and $\mathbb{J}_{1}(\cdot)$ is the Bessel function of the first kind of order one.

\section{Analytical Channel Modeling}

The results of previous works in [16], [17] are provided for a specified case wherein $\sigma_{t x}=\sigma_{t y}, \sigma_{r x}=\sigma_{r y}$, and $\theta_{t x}^{\prime}=$ $\theta_{t y}^{\prime}=\theta_{r x}^{\prime}=\theta_{r y}^{\prime}=0$. In this paper, we consider a general case with non-zero boresight angle wherein the variances of UAV's orientation and position fluctuations are not necessarily equal in the direction of $x$ and $y$ axes, i.e., $\sigma_{t x}, \sigma_{t y}, \sigma_{r x}$, and $\sigma_{r y}$ can take any different values and $\theta_{t x}^{\prime} \neq 0, \theta_{t y}^{\prime} \neq 0, \theta_{r x}^{\prime} \neq 0$, $\theta_{r y}^{\prime} \neq 0$.

Theorem 1. The distribution of link loss induced by AoA fluctuations is derived as

$$
f_{h_{p a}}\left(h_{p a}\right)=\mathbb{R} \delta\left(h_{p a}-1\right)+(1-\mathbb{R}) \delta\left(h_{p a}\right),
$$

where $\mathbb{R}=\sum_{n=1}^{N^{\prime}} \mathbb{R}_{n}$ and

$$
\begin{aligned}
\mathbb{R}_{n} & =\left[Q\left(\frac{-\sqrt{\theta_{\mathrm{FOV}}^{2}-\left(\frac{\theta_{\mathrm{FOV}}}{N^{\prime}}(n-1)\right)^{2}}-\theta_{t x}^{\prime}-\theta_{r x}^{\prime}}{\sqrt{\sigma_{t x o}^{2}+\sigma_{r x o}^{2}}}\right)\right. \\
& \left.-Q\left(\frac{\sqrt{\theta_{\mathrm{FOV}}^{2}-\left(\frac{\theta_{\mathrm{FOV}}}{N^{\prime}}(n-1)\right)^{2}}-\theta_{t x}^{\prime}-\theta_{r x}^{\prime}}{\sqrt{\sigma_{t x o}^{2}+\sigma_{r x o}^{2}}}\right)\right] \\
& {\left[Q\left(\frac{\frac{\theta_{\mathrm{FOV}}}{N^{\prime}}(n-1)-\theta_{t y}^{\prime}-\theta_{r y}^{\prime}}{\sqrt{\sigma_{t y o}^{2}+\sigma_{r y o}^{2}}}\right)\right.} \\
& \left.-Q\left(\frac{\frac{n \theta_{\mathrm{Fov}}}{N^{\prime}}-\theta_{t y}^{\prime}-\theta_{r y}^{\prime}}{\left.\sqrt{\sigma_{t y o}^{2}+\sigma_{r y o}^{2}}\right)+Q}\right) \frac{-\frac{n \theta_{\mathrm{Fov}}}{N^{\prime}}-\theta_{t y}^{\prime}-\theta_{r y}^{\prime}}{\sqrt{\sigma_{t y o}^{2}+\sigma_{r y o}^{2}}}\right) \\
& \left.-Q\left(\frac{-\frac{\theta_{\mathrm{FOV}}}{N^{\prime}}(n-1)-\theta_{t y}^{\prime}-\theta_{r y}^{\prime}}{\sqrt{\sigma_{t y o}^{2}+\sigma_{r y o}^{2}}}\right)\right]
\end{aligned}
$$

where $Q(\cdot)$ and $\delta($.$) are the well-known Q$-function and Dirac delta function, respectively.

Proof: Please refer to Appendix A

In (6), the parameter $N^{\prime}$ is a positive integer and when $N^{\prime}$ grows, the analytical results of (6) leads to the simulation results.

Next, we derive the analytical channel models for UAV-toUAV (UU) FSO links for a wide range of weak to strong atmospheric turbulence conditions.

\section{A. For Weak to Moderate Turbulence Conditions}

Theorem 2. Under weak to moderate atmospheric turbulence conditions, a probability density function (PDF) of the considered UU channel is formulated as

$$
\begin{aligned}
& f_{h}(h)=(1-\mathbb{R}) \delta(h)+\frac{c_{1} w_{z}^{2} \mathbb{R}}{h \sqrt{2 \pi \sigma_{L}^{2}}} \\
& \int_{0}^{2 \pi} \int_{0}^{\infty} x e^{c_{3} x^{2}+c_{2} x} e^{-\frac{\left(\ln \left(\frac{h}{A_{0} h_{l}}\right)+x^{2}-2 \mu_{L}\right)^{2}}{8 \sigma_{L}^{2}}} \mathrm{~d} x \mathrm{~d} \phi,
\end{aligned}
$$




$$
h_{p a}=\frac{1}{\pi} \int_{-r_{a p}}^{r_{a p}} \int_{-\sqrt{r_{a p}^{2}-r^{2}}}^{\sqrt{r_{a p}^{2}-x^{2}}}\left(\frac{\mathbb{J}_{1}\left(\frac{\pi}{\lambda N_{f}} \sqrt{\left(x-d_{f} \tan \left(\theta_{t x}+\theta_{r x}\right)\right)^{2}+\left(y-d_{f} \tan \left(\theta_{t y}+\theta_{r y}\right)\right)^{2}}\right)}{\sqrt{\left(x-d_{f} \tan \left(\theta_{t x}+\theta_{r x}\right)\right)^{2}+\left(y-d_{f} \tan \left(\theta_{t y}+\theta_{r y}\right)\right)^{2}}}\right)^{2} \mathrm{~d} x \mathrm{~d} y .
$$

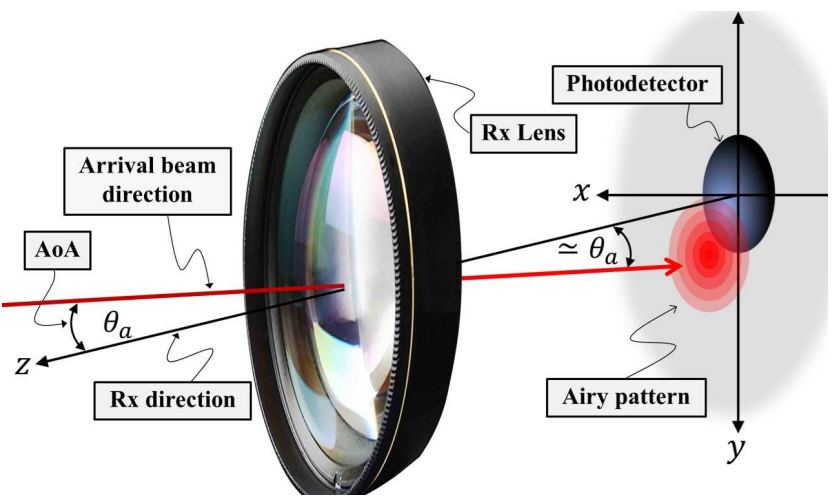

Fig. 1. A schematic of the deviated optical beam due to the AoA fluctuations. The deviated optical beam is focused by a converging lens will compose a deviated Airy pattern at the focal plane.

where

$$
\left\{\begin{array}{l}
c_{1}=\frac{w_{z}^{2}}{8 \pi \sigma_{d x} \sigma_{d y}} \exp \left(-\frac{Z^{2} \theta_{t x}^{\prime 2}}{2 \sigma_{d x}^{2}}-\frac{Z^{2} \theta_{t y}^{\prime 2}}{2 \sigma_{d y}^{2}}\right), \\
c_{2}=\frac{w_{z}}{\sqrt{2}}\left(\frac{Z \theta_{t x}^{\prime} \cos (\phi)}{\sigma_{d x}^{2}}+\frac{Z \theta_{t y}^{\prime} \sin (\phi)}{\sigma_{d y}^{2}}\right), \\
c_{3}=\frac{w_{z}^{2}\left(\sigma_{x}^{2}-\sigma_{y}^{2}\right) \cos (2 \phi)-w_{z}^{2}\left(\sigma_{d x}^{2}+\sigma_{d y}^{2}\right)}{8 \sigma_{d x}^{2} \sigma_{d y}^{2}} .
\end{array}\right.
$$

Proof: Please refer to Appendix B

As we will observe, the proposed channel model in (8) well models the optical channels between UAVs under weak to moderate turbulence condition. However, it consists of a two-dimensional integral. In the next Theorem, we try to find a more tractable channel models under weak turbulence conditions.

Theorem 3. Under weak to moderate atmospheric turbulence conditions, a closed-form PDF of the considered UU link is obtained as

$f_{h}(h)=(1-\mathbb{R}) \delta(h)+v_{3} \mathbb{R} h^{\tau_{1}-1} Q\left(v_{1} \ln \left(\frac{h}{A_{0} h_{l}}\right)-v_{2}\right)$,

where $v_{1}=\frac{1}{2 \sigma_{L}^{2}}, \quad v_{2}=\left(\frac{\mu_{L}}{2 v_{1} \sigma_{L}^{2}}-\frac{\tau_{1}}{v_{1}}\right), \quad v_{3}=$ $\tau_{1}\left(A_{0} h_{l}\right)^{-\tau_{1}} \exp \left(\frac{v_{2}^{2}}{2}-2 \mu_{L}^{2} v_{1}^{2}\right)$, and $\tau_{1}=\frac{w_{z}^{2}}{4 \sigma_{m}^{2}}$.

Proof: Please refer to Appendix [C]

The channel model proposed in (10) is more tractable than (8) and as we will observe in the next Section, (10) is valid over a wide range of pointing errors. However, the proposed channel model in (10) deviates from on which obtained by simulation for a special case wherein $\left[\left(\theta_{t x}^{\prime}+\theta_{r x}^{\prime}\right)^{2}+\left(\theta_{t y}^{\prime}+\right.\right.$ $\left.\left.\theta_{r y}^{\prime}\right)^{2}\right]>\left[9 \max \left(\sigma_{t x o}^{2}+\sigma_{r x o}^{2}, \sigma_{t y o}^{2}+\sigma_{r y o}^{2}\right)\right]$. This deviation is related to the approximation used in (31).

Theorem 4. Under weak to moderate atmospheric turbulence conditions, when the UAV have proximately same $\sigma_{t x o} \simeq \sigma_{t y o}=\sigma_{t o}$ and $\sigma_{r x o} \simeq \sigma_{\text {ryo }}=\sigma_{r o}$, the channel PDF of the considered UU link is formulated as

$$
\begin{aligned}
f_{h}(h) & =\mathbb{M}\left(\frac{\theta_{d}}{\sqrt{\sigma_{\text {to }}^{2}+\sigma_{r o}^{2}}}, \frac{\theta_{\mathrm{FOV}}}{\sqrt{\sigma_{\text {to }}^{2}+\sigma_{\text {ro }}^{2}}}\right) \delta(h) \\
+ & \left(1-\mathbb{M}\left(\frac{\theta_{d}}{\sqrt{\sigma_{\text {to }}^{2}+\sigma_{\text {ro }}^{2}}}, \frac{\theta_{\mathrm{FOV}}}{\sqrt{\sigma_{\text {to }}^{2}+\sigma_{\text {ro }}^{2}}}\right)\right) \times f_{h}(h>0),
\end{aligned}
$$

where

$$
f_{h}(h>0)=\left\{\begin{array}{ll}
f_{h}^{a}(h) & \text { for } \quad h \geq e^{q_{2}} \\
f_{h}^{b}(h) & \text { for } \quad 0<h<e^{q_{2}}
\end{array},\right.
$$

and

$$
\left\{\begin{array}{c}
f_{h}^{a}(h)=q_{3} \frac{e^{\tau \ln h}}{h} \sum_{k=0}^{K} \sum_{j=0}^{k} q_{1}\left(q_{2}-\ln h\right)^{k-j} \\
\times \Gamma\left(\frac{j+1}{2}, \frac{\left(q_{2}-\ln h\right)^{2}}{8 \sigma_{L}^{2}}\right), \\
f_{h}^{b}(h)=q_{3} \frac{e^{\ln h}}{h} \sum_{k=0}^{K} \sum_{j=0}^{k} q_{1}\left(q_{2}-\ln h\right)^{k-j} \\
\times\left[\Gamma\left(\frac{j+1}{2}, 0\right)+(-1)^{j} \Upsilon\left(\frac{j+1}{2}, \frac{\left(q_{2}-\ln h\right)^{2}}{8 \sigma_{L}^{2}}\right)\right],
\end{array}\right.
$$

and the constant $q_{1}, q_{2}$, and $q_{3}$ are

$$
\left\{\begin{array}{l}
q_{1}=\left(\begin{array}{c}
k \\
j
\end{array}\right) \frac{\left(r_{o}^{2} w_{z}^{2} / 8 \sigma_{d}^{4}\right)^{k}\left(8 \sigma_{L}^{2}\right)^{(j+1) / 2}}{\Gamma(k+1) k !}, \\
q_{2}=\ln \kappa h_{l}+2 \mu_{L}-4 \sigma_{L}^{2} \tau \\
q_{3}=\frac{\tau \exp \left(-\tau\left(q_{2}+2 \sigma_{L}^{2} \tau\right)-r_{o}^{2} / 2 \sigma_{d}^{2}\right)}{2 \sqrt{8 \pi} \sigma_{L}} .
\end{array}\right.
$$

Proof: Please refer to Appendix D,

Proposition 1. When $\frac{\sigma_{d}}{r_{o}}>0.8$, (12) can be simplified as

$$
\begin{aligned}
& f_{h>0}(h)=\frac{2 s_{0}}{\sqrt{\pi} h}\left(s_{1}+\frac{s_{2}\left(q_{2}-\ln h\right)}{\sqrt{8 \sigma_{L}^{2}}}\right) \\
& +\frac{s_{0}}{h} \exp \left(\frac{\left(q_{2}-\ln h\right)^{2}}{8 \sigma_{L}^{2}}\right) \operatorname{erfc}\left(-\frac{q_{2}-\ln h}{\sqrt{8 \sigma_{L}^{2}}}\right) \\
& \times\left(2+s_{2}+\frac{2 s_{1}\left(q_{2}-\ln h\right)}{\sqrt{8 \sigma_{L}^{2}}}+\frac{2 s_{2}\left(q_{2}-\ln h\right)^{2}}{8 \sigma_{L}^{2}}\right),
\end{aligned}
$$

where $\operatorname{erfc}($.$) is the well-known complementary error func-$ tion, $r_{o}=Z \sqrt{\theta_{t x}^{\prime 2}+\theta_{t y}^{\prime 2}}, \sigma_{d}^{2}=Z^{2} \sigma_{\mathrm{to}}^{2}+\sigma_{\mathrm{tp}}^{2}+\sigma_{\mathrm{rp}}^{2}, s_{0}=$ $\frac{\tau \exp \left(-r_{o}^{2} / 2 \sigma_{d}^{2}\right)}{4} e^{-\left(\ln \frac{\kappa h_{l}}{h}+2 \mu_{L}\right)^{2} / 8 \sigma_{L}^{2}}, s_{1}=\frac{\sqrt{2} \sigma_{L} r_{r^{2}}^{2} w_{z}^{2}}{4 \sigma_{d}^{4}}$, and $s_{2}=\frac{\sigma_{L}^{2} r_{o}^{4} w_{z}^{4}}{16 \sigma_{d}^{8} \Gamma(3)}$.

Proof: When $\frac{\sigma_{d}}{r_{o}}>0.8$, (40) can be approximated as

$$
\begin{aligned}
f_{h^{\prime}}\left(h^{\prime}\right) & \simeq \frac{\tau e^{-r_{o}^{2} / 2 \sigma_{d}^{2}}}{h^{\prime} \sqrt{8 \pi} \sigma_{L}} \int_{0}^{\infty}\left(1+\frac{r_{o}^{2} w_{z}^{2} x}{8 \sigma_{d}^{4}}+\frac{r_{o}^{4} w_{z}^{4} x^{2}}{256 \sigma_{d}^{8}}\right) \\
& \times \exp \left(-\frac{\left(x-\left(\ln \frac{\kappa h_{l}}{h^{\prime}}+2 \mu_{L}\right)\right)^{2}+8 \sigma_{L}^{2} \tau x}{8 \sigma_{L}^{2}}\right) d x .
\end{aligned}
$$

Using [22, eq. (01.03.21.0104.01)], (35), (16), [17, eq. (21)], and after some mathematical derivations, the closed-form expressions for $f_{h}(h>0)$ is derived in (15). 


\section{B. For Moderate to Strong Turbulence Conditions}

Theorem 5. Under moderate to strong atmospheric turbulence conditions, the channel PDF of the considered UU link is formulated as

$$
\begin{aligned}
f_{h}(h)= & (1-\mathbb{R}) \delta(h)+c_{4} h^{\frac{\alpha+\beta}{2}-1} \int_{0}^{2 \pi} \int_{0}^{A_{0}} x^{-\frac{\alpha+\beta}{2}-c_{3}-1} \\
& \times \mathbb{R} e^{c_{2} \sqrt{\ln \left(\frac{A_{0}}{x}\right)}} k_{\alpha-\beta}\left(\sqrt{\frac{4 \alpha \beta h}{h_{l} x}}\right) \mathrm{d} x \mathrm{~d} \phi
\end{aligned}
$$

where $c_{4}=\frac{2 c_{1} A_{0}^{c_{3}}}{\Gamma(\alpha) \Gamma(\beta)}\left(\frac{\alpha \beta}{h_{l}}\right)^{\frac{\alpha+\beta}{2}}$.

Proof: Please refer to Appendix E

As we will observe in the next Section, the proposed channel model in (17) well models the optical channels between UAVs under moderate to strong turbulence condition. However, it consists of a two-dimensional integral. In the next Theorem, we provide a more tractable channel models under moderate to strong turbulence conditions.

Theorem 6. Under moderate to strong atmospheric turbulence conditions, the closed-form PDF of the considered UU link is formulated as

$$
\begin{aligned}
f_{h}(h)= & (1-\mathbb{R}) \delta(h)+\mathbb{R} \sum_{m=0}^{M}\left[k_{3}\left(\left(A_{0} h_{l} h_{m}\right)^{k_{1}}-h^{k_{1}}\right)\right. \\
& \left.-k_{4}\left(\left(A_{0} h_{l} h_{m}\right)^{k_{2}}-h^{k_{2}}\right)\right] h^{\tau_{1}-1},
\end{aligned}
$$

where $0<h \leq A_{0} h_{l} h_{m}, \nu_{b}=\alpha-\beta$, and

$$
\left\{\begin{aligned}
k_{1} & =m-\tau_{1}+\beta, \quad k_{2}=m-\tau_{1}+\alpha, \\
k_{3} & =\frac{k_{5}(\alpha \beta)^{m-\frac{\nu_{b}}{2}}\left(A_{0} h_{l}\right)^{-k_{1}}}{k_{1} m ! \Gamma\left(m-\nu_{b}+1\right)}, \\
k_{4} & =\frac{k_{5}(\alpha \beta)^{m+\frac{\nu_{b}}{2}}\left(A_{0} h_{l}\right)^{-k_{2}}}{k_{2} m ! \Gamma\left(m+\nu_{b}+1\right)} \\
k_{5} & =\frac{\pi\left(\alpha \beta \frac{\alpha+\beta}{2}\right.}{\Gamma(\alpha) \Gamma(\beta) \sin \left(\pi \nu_{b}\right)\left(A_{0} h_{l}\right)^{\tau_{1}}} .
\end{aligned}\right.
$$

Moreover, the parameters $M$ and $h_{m}$ are given in [17, Table I].

Proof: Please refer to Appendix $\mathrm{F}$

The channel model proposed in (18) is more tractable than (17) and as we will observe in the next Section, (18) is valid over a wide range of pointing errors. However, the proposed channel model in (18) deviates from on which obtained by simulation for a special case wherein $\left[\left(\theta_{t x}^{\prime}+\theta_{r x}^{\prime}\right)^{2}+\left(\theta_{t y}^{\prime}+\right.\right.$ $\left.\left.\theta_{r y}^{\prime}\right)^{2}\right]>\left[9 \max \left(\sigma_{t x o}^{2}+\sigma_{r x o}^{2}, \sigma_{t y o}^{2}+\sigma_{r y o}^{2}\right)\right]$. This deviation is related to the approximation used in (31).

Theorem 7. Under moderate to strong atmospheric turbulence conditions, the channel PDF of considered UU link is formulated as

$$
\begin{aligned}
f_{h}(h) & =\mathbb{M}\left(\frac{\theta_{d}}{\sqrt{\sigma_{\text {to }}^{2}+\sigma_{\text {ro }}^{2}}}, \frac{\theta_{\mathrm{FOV}}}{\sqrt{\sigma_{\text {to }}^{2}+\sigma_{\text {ro }}^{2}}}\right) \delta(h) \\
+ & \left(1-\mathbb{M}\left(\frac{\theta_{d}}{\sqrt{\sigma_{\text {to }}^{2}+\sigma_{\text {ro }}^{2}}}, \frac{\theta_{\mathrm{FOV}}}{\sqrt{\sigma_{\text {to }}^{2}+\sigma_{\text {ro }}^{2}}}\right)\right) \times f_{h}(h>0),
\end{aligned}
$$

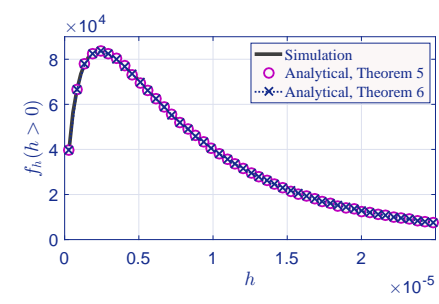

(a)

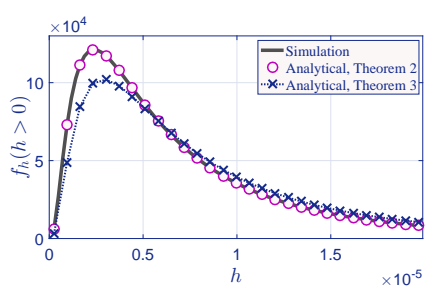

(b)
Fig. 2. Comparison of the accuracy of channel PDFs given in Theorems 2 and 3 when $\sigma_{t x o}=3, \sigma_{t y o}=4, \sigma_{r x o}=3$, and $\sigma_{r y o}=2 \mathrm{mrad}$ and for (a) moderate boresight with $\theta_{t x}^{\prime}=2, \theta_{t y}^{\prime}=3, \theta_{r x}^{\prime}=2$ and $\theta_{r y}^{\prime}=3 \mathrm{mrad}$, and (b) higher boresight with $\theta_{t x}^{\prime}=9, \theta_{t y}^{\prime}=7, \theta_{r x}^{\prime}=5$ and $\theta_{r y}^{\prime}=6 \mathrm{mrad}$

g. 3. Comparison of the accuracy of channel PDFs given in Theorems and 6 when $\sigma_{t x o}=3, \sigma_{t y o}=4, \sigma_{r x o}=3$, and $\sigma_{r y o}=2 \mathrm{mrad}$ and for (a) moderate boresight with $\theta_{t x}^{\prime}=2, \theta_{t y}^{\prime}=3, \theta_{r x}^{\prime}=2$ and $\theta_{r y}^{\prime}=3 \mathrm{mrad}$, and (b) higher boresight with $\theta_{t x}^{\prime}=9, \theta_{t y}^{\prime}=7, \theta_{r x}^{\prime}=5$ and $\theta_{r y}^{\prime}=6 \mathrm{mrad}$.

where

$$
\begin{aligned}
& f_{h}(h>0)=\sum_{m=0}^{M} \sum_{k=0}^{K} \sum_{j=0}^{k} g_{0} g_{1 k}(h)^{\gamma-1}\left(\ln \left(\frac{\kappa h_{l} h_{m}}{h}\right)\right)^{k-j} \\
& +\sum_{m=0}^{M} \sum_{k=0}^{K} g_{1 k}\left(g_{3 m} g_{7 k}(h)^{m+\alpha-1}-g_{2 m} g_{6 k}(h)^{m+\beta-1}\right)
\end{aligned}
$$

and

$$
\left\{\begin{array}{l}
g_{0}=\left(g_{2 m} g_{4 j}-g_{3 m} g_{5 j}\right), \\
g_{1 k}=\frac{\pi \gamma\left(r_{o}^{2} w_{z}^{2} / 8 \sigma_{d}^{4}\right)^{k} \exp \left(-r_{o}^{2} / 2 \sigma_{d}^{2}\right)}{k ! \Gamma(k+1) \Gamma(\alpha) \Gamma(\beta) \sin (\pi(\alpha-\beta))}, \\
g_{2 m}=\frac{\left(\alpha \beta / \kappa h_{l}\right)^{m+\beta}}{\Gamma(m+\beta-\alpha+1) m !}, \quad g_{3 n}=\frac{\left(\alpha \beta / \kappa h_{l}\right)^{m+\alpha}}{\Gamma(m+\alpha-\beta+1) n !}, \\
g_{4 j}=\frac{(-1)^{j} j !\left(\begin{array}{l}
k \\
j
\end{array}\right)\left(\kappa h_{l} h_{m}\right)^{m+\beta-\gamma}}{(m+\beta-\gamma)^{j+1}}, \\
g_{5 j}=\frac{(-1)^{j} j !\left(\begin{array}{l}
k \\
j
\end{array}\right)\left(\kappa h_{l} h_{m}\right)^{m+\alpha-\gamma}}{(m+\alpha-\gamma)^{j+1}}, \\
g_{6 k}=\frac{(-1)^{k} k !}{(n+\beta-\gamma)^{k+1}}, \quad g_{7 k}=\frac{(-1)^{k} k !}{(n+\alpha-\gamma)^{k+1}} .
\end{array}\right.
$$

Proof: Please refer to Appendix $\mathrm{G}$

Remark 1. The channel model provided in this paper are for the general UU link. The results can be used for the special case of the ground-to-UAV link by setting the parameters related to the Tx orientation fluctuations to zero. Similarly, the results can be used for UAV-to-ground link by setting the parameters related to the Rx orientation fluctuations to zero.

\section{Numerical Results}

We utilize computer simulations to verify the accuracy of our proposed analytical channel models for UAV-based FSO 


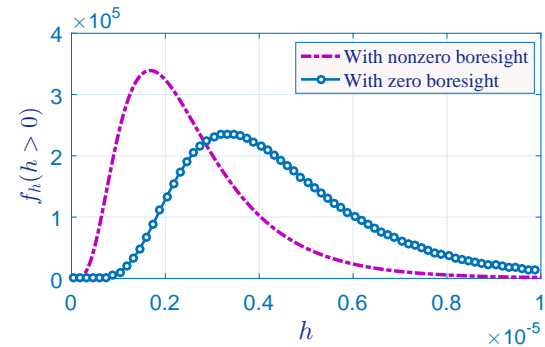

Fig. 4. Comparison of channel PDF of UAV-based FSO links with zero and nonzero boresight for $\sigma_{t o}=\sigma_{r o}=4 \mathrm{mrad}$. For nonzero boresight, $\theta_{i}^{\prime}=8$ $\operatorname{mrad}$ where $i \in\{\mathrm{tx}, \mathrm{ty}, \mathrm{rx}, \mathrm{ry}\}$.

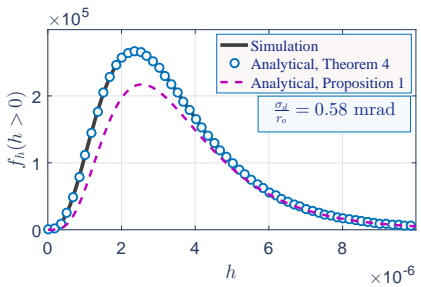

(a)

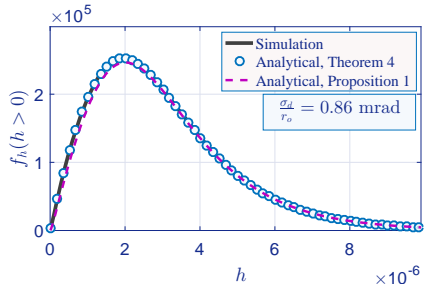

(b)
Fig. 5. Comparison of the accuracy of channel PDFs given in Theorem 4 and Proposition 1 for (a) $\sigma_{t o}=\sigma_{r o}=4 \mathrm{mrad}$, and (b) $\sigma_{t o}=\sigma_{r o}=6 \mathrm{mrad}$.

links. We set the system parameters under simulation as link length $Z=500 \mathrm{~m}$, receiver lens radius $r_{a}=5 \mathrm{~cm}$, Rytov variance for weak turbulence $\sigma_{R}^{2}=0.2$, for strong turbulence $\sigma_{R}^{2}=2$, standard deviation of UAV position $\sigma_{t x p}=\sigma_{r x p}=40$ $\mathrm{cm}, \sigma_{\text {typ }}=\sigma_{\text {ryp }}=30 \mathrm{~cm}, N^{\prime}=10$, and $K=10$. Moreover, the parameters $N$ and $h_{m}$ are given in [17, Table I].

For evaluation of analytical channel models provided in Section III, we perform Monte-Carlo simulations. The details of the simulation process are described as follows. For given $\theta_{i}^{\prime}$ and $\sigma_{i o}$ where $i \in\{t x, t y, r x, r y\}$, we generate $10^{7}$ independent RVs $\theta_{t x}, \theta_{t y}, \theta_{r x}$, and $\theta_{r y}$. Then, based on (5), we generate $10^{7}$ independent coefficients of $h_{p a}$. Moreover, for given $\sigma_{i p}$, we generate $10^{7}$ independent RVs $x_{t x}, y_{t y}, x_{r x}$, and $x_{r y}$. Then, using generated RVs $\theta_{t x}, \theta_{t y}, x_{t x}, x_{t y}, x_{r x}$ and $x_{r y}$, we generate $10^{7}$ independent coefficients of $h_{p g}$ from (3). For a given $\sigma_{R}^{2}<0.5$, we also generate $10^{7}$ independent coefficients of $h_{a}$ which have log-normal distribution as given in (27). For a given $\sigma_{R}^{2}>0.5$, we generate $10^{7}$ independent coefficients of $h_{a}$ which have GG distribution as given in (43). We then obtain $10^{7}$ independent values of UAV-based optical channel coefficients based on (2). Finally, we find the channel distribution diagrams. It is worth mentioning that, for each state of simulation, we perform independent runs in MATLAB which takes about 20 minutes of processing time (Intel Core i7 Processors, 8 GB RAM). On the other hand, by using our proposed analytical-based methods proposed in Section III, the channel can be easily modeled in less than a second which is extremely faster than employing simulation-based methods.

First, in Fig. 2] we corroborate the accuracy of the derived analytical channel model in Theorems 2 and 3. The results of Figs. $2 \mathrm{a}$ and $2 \mathrm{~b}$ are plotted for $\sigma_{t x o}=3, \sigma_{t y o}=4, \sigma_{\text {rxo }}=3$, and $\sigma_{\text {ryo }}=2 \mathrm{mrad}$ and for a wide range of boresight values: (a) moderate boresight with $\theta_{t x}^{\prime}=2, \theta_{t y}^{\prime}=3, \theta_{r x}^{\prime}=2$ and $\theta_{r y}^{\prime}=3 \mathrm{mrad}$, and (b) higher boresight with $\theta_{t x}^{\prime}=$ $9, \theta_{t y}^{\prime}=7, \theta_{r x}^{\prime}=5$ and $\theta_{r y}^{\prime}=6 \mathrm{mrad}$. The results of Figs. $2 \mathrm{a}$ and $2 \mathrm{~b}$ clearly show that the analytical channel model derived in Theorem 2 is valid for all conditions. In Theorem 3 , we also propose a more tractable closed-form channel model. As previously mentioned, the analytical channel model derived in Theorem 3 is accurate over a wide conditions, expect a specific condition wherein $\left[\left(\theta_{t x}^{\prime}+\theta_{r x}^{\prime}\right)^{2}+\left(\theta_{t y}^{\prime}+\right.\right.$ $\left.\left.\theta_{r y}^{\prime}\right)^{2}\right]>\left[9 \max \left(\sigma_{t x o}^{2}+\sigma_{r x o}^{2}, \sigma_{\text {tyo }}^{2}+\sigma_{r y o}^{2}\right)\right]$. The results of Fig. 2a confirm the accuracy of expression given in Theorem 3. However, for the aforementioned specific condition, the analytical channel model given in Theorem 3 deviates from simulation results. Notice, the channel models given in Theorems 2 and 3 are provided for weak to moderate atmospheric turbulence conditions. Similarly, for moderate to strong turbulence conditions, in Fig. 3. we corroborate the accuracy of the derived analytical channel models in Theorems 5 and 6 . The parameter values related to the UAVs' orientation fluctuations of Fig. 3 are equal to the parameter values used in Fig. 2. Simulation results confirm the accuracy of analytical channel model given in Theorem 5. Also, the closed-form channel model derived in Theorem 6 is accurate over wide range of UAVs' orientation fluctuations, expect a specific condition wherein $\left[\left(\theta_{t x}^{\prime}+\theta_{r x}^{\prime}\right)^{2}+\left(\theta_{t y}^{\prime}+\theta_{r y}^{\prime}\right)^{2}\right]>$ $\left[9 \max \left(\sigma_{\text {txo }}^{2}+\sigma_{\text {rxo }}^{2}, \sigma_{\text {tyo }}^{2}+\sigma_{\text {ryo }}^{2}\right)\right]$.

In Fig. 4 we compare the channel distribution of considered UAV-based system with zero and nonzero boresight pointing errors with same $\sigma_{t x o}=\sigma_{t y o}=\sigma_{r x o}=\sigma_{r y o}=4 \mathrm{mrad}$. This figure clearly shows that we can not neglect the effect of boresight pointing errors in UAV-based FSO communications even when $\sigma_{t x o}=\sigma_{t y o}$ and $\sigma_{t x o}=\sigma_{t y o}$, and reveals the importance of Theorems 4 and 7.

In Fig. 5, by employing Monte-Carlo simulations, the accuracy of proposed closed-form channel PDFs under weak to moderate turbulence conditions given in Theorem 4 and Proposition 1, is investigated for two different conditions. The results are obtained for angular boresight $\theta_{i}^{\prime}=5 \mathrm{mrad}$ where $i \in\{\mathrm{tx}, \mathrm{ty}, \mathrm{rx}, \mathrm{ry}\}$. The results of Fig. 5] confirm the validity of analytical channel PDF proposed in Theorem 4. A more simpler channel PDF is also proposed in Proposition 1. As proven and demonstrated in Fig. 5, the simpler channel model is valid when $\frac{\sigma_{d}}{r_{d}}>0.8$. In Theorem 7 , we proposed a channel model that is suitable for moderate to strong turbulence conditions. The results of Fig. 6 confirm the accuracy of the proposed channel PDF.

\section{CONCLUSION}

In this paper, we proposed comprehensive and novel channel models for UAV-based FSO links that takes into account the effects of nonzero boresight pointing errors along with the effects of UAVs' orientation and position fluctuations, atmospheric turbulence strength, optical beamwidth, link length, lens radius size, receiver's FOV, etc. In addition to the tractability, simulation results confirm the accuracy of the proposed analytical channel models. To assess the benefits of UAV-based FSO deployments, the proposed channel models 


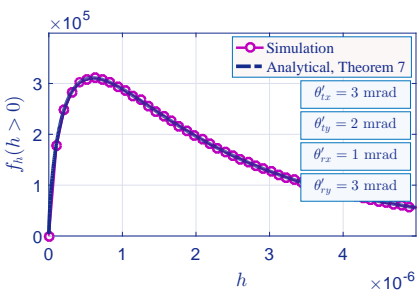

(a)

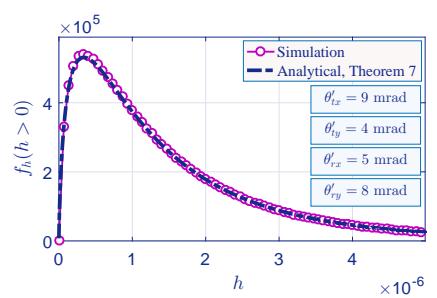

(b)
Fig. 6. Comparison of the accuracy of channel PDFs given in Theorem 7 when $\sigma_{t o}=\sigma_{r o}=5 \mathrm{mrad}$ and for (a) low boresight, and (b) higher boresight.

will assist researchers to easily analyze and design of such systems without using any time-consuming simulations.

\section{APPENDIX A}

\section{PROOF OF THEOREM 1}

Since the AoA angle is in the order of mrad, we can well approximate (4) as

$$
\theta_{a} \simeq \sqrt{\left(\theta_{t x}+\theta_{r x}\right)^{2}+\left(\theta_{t y}+\theta_{r y}\right)^{2}} .
$$

We consider a nonzero boresight error for AoA, and model $\theta_{t x}, \theta_{t y}, \theta_{r x}$, and $\theta_{r y}$ as nonzero mean Gaussian distributed RVs. Hence, from (23), the angle $\theta_{a}$ follows the Beckmann distribution [23]

$$
\begin{aligned}
f_{\theta_{a}}\left(\theta_{a}\right) & =\frac{\theta_{a}}{2 \pi \sqrt{\left(\sigma_{t x o}^{2}+\sigma_{r x o}^{2}\right)\left(\sigma_{t y o}^{2}+\sigma_{r y o}^{2}\right)}} \\
\times \int_{0}^{2 \pi} e^{-\frac{\left(\theta_{a} \cos (\phi)-\theta_{t x}^{\prime}-\theta_{r x}^{\prime}\right)^{2}}{2\left(\sigma_{t x o}^{2}+\sigma_{r x o}^{2}\right)}-\frac{\left(\theta_{a} \sin (\phi)-\theta_{t y}^{\prime}-\theta_{r y}^{\prime}\right)^{2}}{2\left(\sigma_{t y o}^{2}+\sigma_{r y o}^{2}\right)}} & \mathrm{d} \phi .
\end{aligned}
$$

As discussed in Section II, the AoA fluctuations of the hovering lightweight UAVs is in the order of several mrad which is much greater than the optical ground links. To compensate the greater AoA fluctuations, the detector area of the Rx must be selected greater than the detector area of the ground optical links, which makes a greater FoV. From, the results of [17], for a large value of FOV, one can approximate (5) as

$$
h_{p a}= \begin{cases}1 & \text { for } \theta_{a}<\theta_{\mathrm{FOV}} \\ 0 & \text { for } \theta_{a} \geq \theta_{\mathrm{FOV}} .\end{cases}
$$

where $\theta_{\mathrm{FOV}}$ is the receiver's FOV. Now, from (24) and 23, we have

$$
\begin{aligned}
& f_{h_{p a}}\left(h_{p a}\right)=\frac{\delta\left(h_{p a}-1\right)}{2 \pi \sqrt{\left(\sigma_{t x o}^{2}+\sigma_{r x o}^{2}\right)\left(\sigma_{t y o}^{2}+\sigma_{r y o}^{2}\right)}} \times \\
& \int_{0}^{\theta_{\mathrm{FOV}}} \int_{0}^{2 \pi} \theta_{a} e^{-\frac{\left(\theta_{a} \cos (\phi)-\theta_{t x}^{\prime}-\theta_{r x}^{\prime}\right)^{2}}{2\left(\sigma_{t x o}^{2}+\sigma_{r x o}^{2}\right)}-\frac{\left(\theta_{a} \sin (\phi)-\theta_{t y}^{\prime}-\theta_{r y}^{\prime}\right)^{2}}{2\left(\sigma_{t y o}^{2}+\sigma_{r y o}^{2}\right)}} \mathrm{d} \phi \mathrm{d} \theta_{a} \\
& +\delta\left(h_{p a}\right)\left[1-\frac{1}{2 \pi \sqrt{\left(\sigma_{t x o}^{2}+\sigma_{r x o}^{2}\right)\left(\sigma_{t y o}^{2}+\sigma_{r y o}^{2}\right)}} \times\right. \\
& \left.\int_{\theta_{\mathrm{FOV}}}^{\infty} \int_{0}^{2 \pi} \theta_{a} e^{-\frac{\left(\theta_{a} \cos (\phi)-\theta_{t x}^{\prime}-\theta_{r x}^{\prime}\right)^{2}}{2\left(\sigma_{t x o}^{2}+\sigma_{r x o}^{2}\right)}-\frac{\left(\theta_{a} \sin (\phi)-\theta_{t y}^{\prime}-\theta_{r y}^{\prime}\right)^{2}}{2\left(\sigma_{t y o}^{2}+\sigma_{r y o}^{2}\right)}} \mathrm{d} \phi \mathrm{d} \theta_{a}\right] .
\end{aligned}
$$

Similar to the method exploited in [24] and after some manipulations, $f_{h_{p a}}\left(h_{p a}\right)$ is derived in (6).

\section{APPENDIX B \\ PROF OF THEOREM 2}

For weak to moderate atmospheric turbulence conditions, $h_{a}$ can be well modeled by log-normal distribution as

$$
f_{\mathrm{L}}\left(h_{a}\right)=\frac{1}{2 h_{a} \sigma_{L} \sqrt{2 \pi}} \exp \left(-\frac{\left(\ln \left(h_{a}\right)-2 \mu_{L}\right)^{2}}{8 \sigma_{L}^{2}}\right),
$$

where $\sigma_{L}^{2}$ and $\mu_{L}=-\sigma_{L}^{2}$ denote the variance and mean of logirradiance, respectively, where $\sigma_{L}^{2} \simeq \sigma_{R}^{2} / 4$ with $\sigma_{R}^{2}$ being the Rytov variance. From the results of [16], to reduce the effects of Tx's orientation fluctuations, the divergence angle must be selected larger than the one used in conventional terrestrial FSO communications. According to this, for UAV-based FSO communications, (3) can be well approximated as

$$
\begin{aligned}
h_{p g} & \simeq \frac{2 r_{a}^{2}}{w_{z}^{2}} \\
& \times \exp \left(-2 \frac{\left(Z \theta_{t x}+x_{t}+x_{r}\right)^{2}+\left(Z \theta_{t y}+y_{t}+y_{r}\right)^{2}}{w_{z}^{2}}\right)
\end{aligned}
$$

where $r_{a}$ is radius of receiver lens, $w_{z}=$ $w_{0} \sqrt{1+\left(1+\frac{2 w_{0}^{2}}{\left(0.55 C_{n}^{2} k^{2} z\right)^{-6 / 5}}\right)\left(\frac{\lambda z}{\pi w_{0}^{2}}\right)^{2}}$ is optical beamwidth at $\mathrm{Rx}, w_{0}$ is optical beamwidth at $\mathrm{Tx}, C_{n}^{2}$ is the index of refraction structure parameter, $\lambda$ is the optical wave length, and $k$ is the optical wave number. Since the orientation fluctuations of UAV's is in the order of mrad, we can well approximate (1) as $r_{d} \simeq \sqrt{r_{d x}^{2}+r_{d y}^{2}}$ where $r_{d x}=\theta_{t x} Z+x_{t x}+x_{r x}$ and $r_{d y}=\theta_{t y} Z+x_{t y}+x_{r y}$ which have Gaussian distribution as $r_{d x} \sim \mathcal{N}\left(Z \theta_{t x}^{\prime}, \sigma_{r x}^{2}\right)$ and $r_{d y} \sim \mathcal{N}\left(Z \theta_{t y}^{\prime}, Z^{2} \sigma_{\text {tyo }}^{2}+\sigma_{\text {typ }}^{2}+\sigma_{\text {ryp }}^{2}\right)$ where $\sigma_{d x}^{2}=Z^{2} \sigma_{t x o}^{2}+\sigma_{t x p}^{2}+\sigma_{r x p}^{2}$ and $\sigma_{d y}^{2}=Z^{2} \sigma_{t y o}^{2}+\sigma_{t y p}^{2}+\sigma_{r y p}^{2}$. From this, the RV $r_{d}$ follows the Beckmann distribution [23]

$$
\begin{aligned}
& f_{r_{d}}\left(r_{d}\right)= \\
& \quad \frac{r_{d}}{2 \pi \sigma_{d x} \sigma_{d y}} \int_{0}^{2 \pi} e^{-\frac{\left(r_{d} \cos (\phi)-Z \theta_{t x}^{\prime}\right)^{2}}{2 \sigma_{d x}^{2}}-\frac{\left(r_{d} \sin (\phi)-Z \theta_{t y}^{\prime}\right)^{2}}{2 \sigma_{d y}^{2}}} \mathrm{~d} \phi .
\end{aligned}
$$

From (28) and 29), we obtain

$$
\begin{aligned}
& f_{h_{p g}}\left(h_{p g}\right)= \\
& \int_{0}^{2 \pi} \frac{c_{1}}{h_{p g}} \exp \left(c_{3} \ln \left(\frac{A_{0}}{h_{p g}}\right)+c_{2} \sqrt{\ln \left(\frac{A_{0}}{h_{p g}}\right)}\right) \mathrm{d} \phi,
\end{aligned}
$$

where $0<h_{p g} \leq A_{0}$, the parameters $c_{1}, c_{2}$, and $c_{3}$ are obtained in (9) and $A_{0}=\frac{2 r_{a}^{2}}{w_{z}^{2}}$. Finally, from (2), (6), (27) and (30), and after some manipulations, the optical channel model under weak turbulence conditions, is derived in (8).

\section{APPENDIX C \\ PROF OF THEOREM 3}

An approximation for Beckmann distribution is given in [25]. From [25], we can approximate (29] as

$$
f_{r_{d}}\left(r_{d}\right)=\frac{r_{d}}{\sigma_{m}^{2}} \exp \left(-\frac{r_{d}^{2}}{2 \sigma_{m}^{2}}\right)
$$


where

$$
\sigma_{m}^{2}=\left(\frac{3 Z^{2} \theta_{t x}^{\prime 2} \sigma_{d x}^{4}+3 Z^{2} \theta_{t y}^{\prime 2} \sigma_{d y}^{4}+\sigma_{d x}^{6}+\sigma_{d y}^{6}}{2}\right)^{\frac{1}{3}} .
$$

From (28) and (31), we obtain

$$
f_{h_{p g}}\left(h_{p g}\right)=\frac{w_{z}^{2}}{4 \sigma_{m}^{2}} A_{0}^{\frac{4 \sigma_{m}^{2}}{w w_{z}^{2}}} h_{p g}^{\frac{w_{z}^{2}}{4 \sigma_{m}^{2}}-1} . \quad 0 \leq h_{p g} \leq A_{0},
$$

Finally, from (2), (27) and (33), and after some derivations, the closed-form channel model under weak turbulence condition is derived in (10).

\section{APPENDIX D \\ PROF OF THEOREM 4}

In some scenarios, UAVs have approximately same UAV's instability in the $x$ and $y$ axis. Under such conditions, we have $\sigma_{\text {txo }} \simeq \sigma_{\text {tyo }}=\sigma_{t o}, \sigma_{\text {rxo }} \simeq \sigma_{\text {ryo }}=\sigma_{r o}, \sigma_{t x p} \simeq \sigma_{\text {typ }}=\sigma_{t p}$, and $\sigma_{r x p} \simeq \sigma_{r y p}=\sigma_{r p}$, and the AoA of the received signal follows a Rician distribution as

$$
f_{\theta_{a}}\left(\theta_{a}\right)=\frac{\theta_{a}}{\sigma_{t o}^{2}+\sigma_{r o}^{2}} e^{-\frac{\theta_{a}^{2}+\theta_{d}^{2}}{2\left(\sigma_{t_{o}}^{2}+\sigma_{r o}^{2}\right)}} I_{0}\left(\frac{\theta_{a} \theta_{d}}{\sigma_{t_{o}}^{2}+\sigma_{r o}^{2}}\right),
$$

where $I_{0}($.$) is the modified Bessel function of the$ first kind with order zero, $\theta_{a} \in[0, \infty)$, and $\theta_{d}=$ $\sqrt{\left(\theta_{t x}^{\prime}+\theta_{r x}^{\prime}\right)^{2}+\left(\theta_{t y}^{\prime}+\theta_{r y}^{\prime}\right)^{2}}$ is the boresight angle of received beam. From (25) and (34), we have

$$
\begin{array}{r}
f_{h_{p a}}\left(h_{p a}\right)=\mathbb{M}\left(\frac{\theta_{d}}{\sqrt{\sigma_{\text {to }}^{2}+\sigma_{r o}^{2}}}, \frac{\theta_{\mathrm{FOV}}}{\sqrt{\sigma_{\text {to }}^{2}+\sigma_{r o}^{2}}}\right) \delta\left(h_{p a}\right) \\
+\left(1-\mathbb{M}\left(\frac{\theta_{d}}{\sqrt{\sigma_{\text {to }}^{2}+\sigma_{\text {ro }}^{2}}}, \frac{\theta_{\mathrm{FOV}}}{\sqrt{\sigma_{\text {to }}^{2}+\sigma_{r o}^{2}}}\right)\right) \delta\left(h_{p a}-1\right),
\end{array}
$$

where $\mathbb{M}(a, b)$ is the Marcum $Q$-function that is represented as [26]

$$
\mathbb{M}(a, b)=\int_{b}^{\infty} x \exp \left(-\frac{x^{2}+a^{2}}{2}\right) I_{0}(a x) .
$$

Note that Marcum $Q$-function is a standard function that is available in popular mathematical software packages, e.g., MATLAB, and Mathematica.

From (28) and after some mathematical calculations, we obtain

$$
\begin{aligned}
& f_{h_{p g}}\left(h_{p g}\right)=\tau\left(\frac{w_{z}^{2}}{2 r_{a}^{2}}\right)^{\tau} \exp \left(-\frac{Z^{2}\left(\theta_{t x}^{\prime 2}+\theta_{t y}^{\prime 2}\right)}{2\left(Z^{2} \sigma_{\mathrm{to}}^{2}+\sigma_{\mathrm{tp}}^{2}+\sigma_{\mathrm{rp}}^{2}\right)}\right) \\
& \times h_{p g}^{\tau-1} I_{0}\left(\sqrt{\frac{Z^{2} w_{z}^{2}\left(\theta_{t x}^{\prime 2}+\theta_{t y}^{\prime 2}\right) \ln \left(\frac{w_{z}^{2}}{2 r_{a}^{2} h_{p g}}\right)}{2\left(Z^{2} \sigma_{\mathrm{to}}^{2}+\sigma_{\mathrm{tp}}^{2}+\sigma_{\mathrm{rp}}^{2}\right)^{2}}}\right),
\end{aligned}
$$

where $h_{p g} \in\left[0,2 r_{a}^{2} / w_{z}^{2}\right]$ and $\tau=\frac{w_{z}^{2}}{4\left(Z^{2} \sigma_{\text {to }}^{2}+\sigma_{\text {tp }}^{2}+\sigma_{\text {tp }}^{2}\right)}$. Let us define $h^{\prime}=h_{l} h_{a} h_{p g}$. The distribution of $h^{\prime}$ is obtained as

$$
f_{h^{\prime}}\left(h^{\prime}\right)=\int \frac{1}{h_{l} h_{a}} f_{h_{p g}}\left(h^{\prime} / h_{l} h_{a}\right) f_{h_{a}}\left(h_{a}\right) d h_{a} .
$$

Substituting (27) and (37) in (38), and after some simplifications, we obtain

$$
\begin{aligned}
f_{h^{\prime}}\left(h^{\prime}\right) & =\frac{\tau \exp \left(-r_{o}^{2} / 2 \sigma_{d}^{2}\right)}{\sqrt{8 \pi} \sigma_{L}}\left(h^{\prime}\right)^{-1} \int_{0}^{\infty} e^{-\tau x} \\
& \times I_{0}\left(\sqrt{\frac{r_{o}^{2} w_{z}^{2} x}{2 \sigma_{d}^{4}}}\right) \exp \left(-\frac{\left(x-\ln \frac{\kappa h_{l}}{h^{\prime}}-2 \mu_{L}\right)^{2}}{8 \sigma_{L}^{2}}\right) d x
\end{aligned}
$$

where $\kappa=\frac{2 r_{a}^{2}}{w_{z}^{2}}, r_{o}=Z \sqrt{\theta_{t x}^{\prime 2}+\theta_{t y}^{\prime 2}}, \sigma_{d}^{2}=Z^{2} \sigma_{\mathrm{to}}^{2}+\sigma_{\mathrm{tp}}^{2}+\sigma_{\mathrm{rp}}^{2}$. Using the identity $I_{\nu}(z)=\sum_{k=0}^{K} \frac{1}{\Gamma(k+\nu+1) k !}\left(\frac{z}{2}\right)^{2 k+\nu}$ [22, eq. (03.02.02.0001.01)], 39) can be represented as

$$
\begin{aligned}
f_{h^{\prime}}\left(h^{\prime}\right) & =\frac{\tau e^{-r_{o}^{2} / 2 \sigma_{d}^{2}}}{h^{\prime} \sqrt{8 \pi} \sigma_{L}} \sum_{k=0}^{K} \frac{\left(r_{o}^{2} w_{z}^{2} / 8 \sigma_{d}^{4}\right)^{k}}{\Gamma(k+1) k !} \int_{0}^{\infty} x^{k} \\
& \times \exp \left(-\frac{\left(x-\left(\ln \frac{\kappa h_{l}}{h^{\prime}}+2 \mu_{L}\right)\right)^{2}+8 \sigma_{L}^{2} \tau x}{8 \sigma_{L}^{2}}\right) d x,
\end{aligned}
$$

where $\Gamma($.$) is the Gamma function. In the following$ derivations we use the upper incomplete Gamma function $\Gamma(s, x)=\int_{x}^{\infty} t^{s-1} e^{-t} d t$ and lower incomplete Gamma function $\Upsilon(s, x)=\int_{0}^{\infty} t^{s-1} e^{-t} d t$ that are supported by MATLAB and Mathematica software packages. Using these and after some manipulations, when $h^{\prime} \geq e^{q_{2}}$, the closed form expression for (40) is obtained as

$$
\begin{aligned}
f_{h^{\prime}}\left(h^{\prime}\right)= & q_{3} \frac{e^{\tau \ln h^{\prime}}}{h^{\prime}} \sum_{k=0}^{K} \sum_{j=0}^{k} q_{1}\left(q_{2}-\ln h^{\prime}\right)^{k-j} \\
& \times \Gamma\left(\frac{j+1}{2}, \frac{\left(q_{2}-\ln h^{\prime}\right)^{2}}{8 \sigma_{L}^{2}}\right),
\end{aligned}
$$

where the constant $q_{1}, q_{2}$, and $q_{3}$ are given in (14). Moreover, when $h^{\prime}<e^{q_{2}}$, the closed form expression for (40) is obtained as

$$
\begin{aligned}
f_{h^{\prime}}\left(h^{\prime}\right) & =q_{3} \frac{e^{\tau \ln h^{\prime}}}{h^{\prime}} \sum_{k=0}^{K} \sum_{j=0}^{k} q_{1}\left(q_{2}-\ln h^{\prime}\right)^{k-j} \\
\times & {\left[\Gamma\left(\frac{j+1}{2}, 0\right)+(-1)^{j} \Upsilon\left(\frac{j+1}{2}, \frac{\left(q_{2}-\ln h^{\prime}\right)^{2}}{8 \sigma_{L}^{2}}\right)\right] . }
\end{aligned}
$$

Finally, using (35), (41), (42), and [17, eq. (21)], the channel $\mathrm{PDF}$ is derived in (11).

\section{APPENDIX E \\ PROF OF THEOREM 5}

For moderate to strong atmospheric turbulence conditions, $h_{a}$ can be well modeled by GG distribution as

$$
f_{\mathrm{G}}\left(h_{a}\right)=\frac{2(\alpha \beta)^{\frac{\alpha+\beta}{2}}}{\Gamma(\alpha) \Gamma(\beta)} h_{a}^{\frac{\alpha+\beta}{2}-1} k_{\alpha-\beta}\left(2 \sqrt{\alpha \beta h_{a}}\right),
$$

where $\beta$ and $\alpha$ are, respectively, the effective number of smallscale and large-scale eddies, which depend on Rytov variance $\sigma_{R}^{2}$, and $k_{\nu}($.$) is the modified Bessel function of the second$ kind of order $\nu$. Based on (2), (6), (30) and (43), and after some manipulations, the optical channel model under weak turbulence conditions, is derived in (17). 


\section{APPENDIX F \\ PROF OF THEOREM 6}

In the following derivation, we use the integral identity

$$
\begin{aligned}
& k_{w}(z)= \\
& \frac{\pi}{2 \sin (\pi w)} \sum_{m=0}^{M}\left[\frac{(z / 2)^{2 m-w}}{\Gamma(m-w+1) m !}-\frac{(z / 2)^{2 m+w}}{\Gamma(m+w+1) m !}\right] .
\end{aligned}
$$

Based on (2), (6), (30), substituting (44) in (43), using the results of [17, Appendix $\mathrm{C}]$, and after some manipulations, the optical channel model under moderate to strong turbulence conditions, is derived in (18).

\section{APPENDIX G \\ PROF OF THEOREM 7}

Substituting (37) and (43) in (38), using (44), applying a change of variable rule $y=\ln \frac{h^{\prime}}{\kappa h_{l} h_{a}}$, and after some manipulations, we obtain

$$
\begin{aligned}
& f_{h^{\prime}}\left(h^{\prime}\right)=B_{0} \sum_{m=0}^{M} h^{\prime m-1} \\
& \times\left[g_{2 m} h^{\prime \beta} \int_{\ln \left(h^{\prime} / \kappa h_{l} h_{a}\right)}^{\infty} e^{(\tau-m-\beta) y} I_{0}\left(\frac{r_{o}}{\sigma_{d}^{2}} \sqrt{\frac{-w_{z}^{2} y}{2}}\right) d y\right. \\
& \left.-g_{3 m} h^{\prime \alpha} \int_{\ln \left(h^{\prime} / \kappa h_{l} h_{a}\right)}^{\infty} e^{(\tau-n-\alpha) y} I_{0}\left(\frac{r_{o}}{\sigma_{d}^{2}} \sqrt{\frac{-w_{z}^{2} y}{2}}\right) d y\right]
\end{aligned}
$$

where $B_{0}=\frac{\pi \tau \exp \left(-r_{o}^{2} / 2 \sigma_{d}^{2}\right)}{\Gamma(\alpha) \Gamma(\beta) \sin (\pi(\alpha-\beta))}$. In the following derivation, we utilize the identity [27, eq. (2.32.2)]

$$
\int e^{a x} x^{k} d x=e^{a x}\left(\sum_{j=0}^{k} \frac{(-1)^{j} j !\left(\begin{array}{c}
k \\
j
\end{array}\right)}{a^{j+1}} x^{k-j}\right) .
$$

Finally, using (35), 45], (46), [22, eq. (03.02.02.0001.01)], [17, eq. (21)], and after some mathematical manipulations, the channel PDF is derived in (20).

\section{REFERENCES}

[1] M. Alzenad, M. Z. Shakir, H. Yanikomeroglu, and M.-S. Alouini, "FSO-based vertical backhaul/fronthaul framework for $5 \mathrm{G}+$ wireless networks," IEEE Commun. Mag., vol. 56, no. 1, pp. 218-224, Jan. 2018.

[2] W. Fawaz, C. Abou-Rjeily, and C. Assi, "UAV-aided cooperation for FSO communication systems," IEEE Commun. Mag., vol. 56, no. 1, pp. 70-75, Jan. 2018.

[3] C. Yan, L. Fu, J. Zhang, and J. Wang, "A comprehensive survey on UAV communication channel modeling," IEEE Access, vol. 7, pp. 107769 $107792,2019$.

[4] A. A. Farid and S. Hranilovic, "Outage capacity optimization for freespace optical links with pointing errors," J. Lightw. Technol., vol. 25, no. 7, pp. 1702-1710, 2007.

[5] A. Jurado-Navas, J. M. Garrido-Balsells, J. F. Paris, and A. PuertaNotario, "A unifying statistical model for atmospheric optical scintillation," arXiv preprint arXiv:1102.1915 2011.
[6] J. M. Garrido-Balsells, A. Jurado-Navas, J. F. Paris, M. Castillo-Vazquez, and A. Puerta-Notario, "Novel formulation of the $\mathcal{M}$ model through the Generalized-K distribution for atmospheric optical channels," Optics express, vol. 23, no. 5, pp. 6345-6358, 2015.

[7] F. Yang, J. Cheng, and T. A. Tsiftsis, "Free-space optical communication with nonzero boresight pointing errors," IEEE Trans. Commun., vol. 62 , no. 2, pp. 713-725, 2014.

[8] V. V. Mai and H. Kimm, "Beam size optimization and adaptation for high-altitude airborne free-space optical communication systems," IEEE Photonics Journal, vol. 11, no. 2, pp. 1-13, 2019.

[9] V. V. Mai and H. Kim, "Adaptive beam control techniques for airborne free-space optical communication systems," Applied optics, vol. 57, no. 26, pp. 7462-7471, 2018 .

[10] H. Safi, A. Dargahi, and J. Cheng, "Spatial beam tracking and data detection for an FSO link to a UAV in the presence of hovering fluctuations," arXiv preprint arXiv:1904.03774 2019.

[11] M. T. Dabiri and S. M. S. Sadough, "Optimal placement of UAVassisted free-space optical communication systems with DF relaying," IEEE Commun. Let., vol. 24, no. 1, pp. 155-158, 2020.

[12] A. Kaadan, H. H. Refai, and P. G. LoPresti, "Multielement FSO transceivers alignment for inter-UAV communications," J. Lightw. Technol., vol. 32, no. 24, pp. 4183-4193, 2014.

[13] A. Kaadan, H. Refai, and P. Lopresti, "Spherical FSO receivers for UAV communication: geometric coverage models," IEEE Trans. Aerosp. Electron. Syst., vol. 52, no. 5, pp. 2157-2167, 2016.

[14] M. Najafi, H. Ajam, V. Jamali, P. D. Diamantoulakis, G. K. Karagiannidis, and R. Schober, "Statistical modeling of FSO fronthaul channel for drone-based networks," in 2018 IEEE International Conference on Communications (ICC). IEEE, May. 2018, pp. 1-7.

[15] _ _ "Statistical modeling of the FSO fronthaul channel for UAV-based networks," arXiv preprint arXiv:1905.12424 2019.

[16] M. T. Dabiri, S. M. S. Sadough, and M. A. Khalighi, "Channel modeling and parameter optimization for hovering UAV-based free-space optical links," IEEE J. Sel. Areas Commun., vol. 36, no. 9, pp. 2104-2113, Sep. 2018.

[17] M. T. Dabiri, S. M. S. Sadough, and I. S. Ansari, "Tractable optical channel modeling between UAVs," IEEE Trans. Veh. Technol., vol. 68 , no. 12, pp. 11 543-11 550, 2019.

[18] M. T. Dabiri, H. Safi, S. Parsaeefard, and W. Saad, "Analytical channel models for millimeter wave UAV networks under hovering fluctuations," IEEE Trans. Wireless Commun., vol. 19, no. 4, pp. 2868-2883, 2020.

[19] M. T. Dabiri, M. Rezaee, B. Maham, W. Saad, and C. S. Hong, "3D channel characterization and performance analysis of UAV-assisted millimeter wave links," arXiv preprint arXiv:2003.00526. 2020.

[20] R. Gagliardi and S. Karp, "Optical communications,(john wiley \& sons)," 1995.

[21] M. Born and E. Wolf, Principles of optics: electromagnetic theory of propagation, interference and diffraction of light. Elsevier, 2013.

[22] Wolfram, "The wolfram functions site: http://functions.wolfram.com/," 2001.

[23] P. Beckmann and A. Spizzichino, "The scattering of electromagnetic waves from rough surfaces," Norwood, MA, Artech House, Inc., 1987, 511 p., 1987.

[24] B. Zhu, Z. Zeng, J. Cheng, and N. C. Beaulieu, "On the distribution function of the generalized Beckmann random variable and its applications in communications," IEEE Trans. Commun., vol. 66, no. 5, pp 2235-2250, 2017.

[25] R. Boluda-Ruiz, A. García-Zambrana, C. Castillo-Vázquez, and B. Castillo-Vázquez, "Novel approximation of misalignment fading modeled by Beckmann distribution on free-space optical links," Optics express, vol. 24, no. 20, pp. 22 635-22 649, 2016.

[26] E. W. Weisstein, "Marcum Q-Function." From MathWorld-A Wolfram Web Resource. http://mathworld.wolfram.com/MarcumQ-Function.html

[27] I. S. Gradshteyn and I. M. Ryzhik, Table of integrals, series, and products. 7th ed. Academic press, 2007. 